\title{
Prediction of Breast Cancer by Segmenting the Image from Mammography using Neural Network Classifier
}

\author{
P.Suganya, Sowmya Ramanathan, Prachi, CHVN Anirudh, A.Yogendra Reddy
}

\begin{abstract}
An automated identification system to enable early identification of breast cancer which is one of the most familiartypes of cancer amidst females which is identified using a diagnostic technique called mammography. This identification ideologybanks on multiple instance learning (MIL) paradigms which demonstrate an aid in therapeutical assistance. Within the projected framework, breasts area unit is first divided adaptively into regions. The GLCM options are extracted from wavelet sub bands. A classification of diagnostic examination procedure as normal or abnormal is revealed from lesions which are masses or small calcifications and the textural options. To arrive at the final results the abovefactors are interpreted from all parts and analysed.In the event of an anomaly found in thereport, the parts that are detected by the machine driven identification will be displayed. Dual evaluation methodology is undertaken to outline this deviation. A neural network has to be trained before utilization; it is done by segmenting the lesions and feeding it to NN. The NN assigns an anomaly index to them and then the combination of local and global anomaly index takes place.
\end{abstract}

Keywords: Multiple instance learning (MIL); diagnostic procedure; mammography; GLCM; computer aided diagnosis (CAD); gray scale image.

\section{INTRODUCTION}

Rapid growth of a bunch of cells within a specific area in the human body is implied as cancer. Tumours can be identified as lumps, distortions or small calcifications which are formed due combining capacity of a bunch of speedily multiplying cells. When harmful tumours are developed from breast cells it is known as breast cancer. The most important historically identified mortality rate in women has been breast cancer.

Revised Manuscript Received on October 18, 2019

Ms.P.Suganya Assistant Professor (O.G), Department of Computer Science and Engineering, Ramapuram Campus, SRM Institute of Science and Technology.

Ms. Sowmya Ramanathan, Student, Department of Computer Science and Engineering, Ramapuram Campus, SRM Institute of Science and Technology.

Ms. Prachi, Student, Department of Computer Science and Engineering, Ramapuram Campus, SRM Institute of Science and Technology

Mr. CHVN Anirudh, Student, Department of Computer Science and Engineering, Ramapuram Campus, SRM Institute of Science and Technology

Mr. A.Yogendra Reddy, Student, Department of Computer Science and Engineering, Ramapuram Campus, SRM Institute of Science and Technology
The vulnerability to breast cancer mortality rates are coming down thanks to the availability of more identification facilities and further leading to fruitful treatments. Mammography which is a diagnostic procedure is the best way of identifying breast cancer in women. Women who are well get x-ray imaging done to discover unexpected breast cancer.

This diagnostic procedure will help in spotting of breast cancer in early stages and aiding in better treatments for survival. Sadly, this is vulnerable for some peculiarities in detection due to human intervention error.This leads to higher unexpected expenditure and also creates a lot of stress and anxiety in the minds of patients. The drawbacks related to errors are due to the misclassification of mammograms.

This is owing to the actual fact that false negatives is an enormous drawback in screening diagnostic procedure as early detection will scale back treatment price, beneficial effects.Faulty misconceptionshave associate degree effect on all the above mentioned parameters as early detection isn't an associate degree choicewith an incorrect identification. Key reason for the probable errors is that the radiologistsdepend upon visual review to be able to take a decision. There is a huge dependency on the radiologists to check the patient's report and arrive at a conclusion. Factors such asexhaustion and repetition of the same task may lead in their interpretation being wrong.

To set right these effects, reimaging mammographic screening is important. It is important to ensure screening of mammograms is done through a machine interface like a machine driven screening or a computer based identification (CAD). The diagnosis majorly utilizes classifier systems. The most important part of this diagnosis is the information sourced by patients to the human specialists but the knowledgeable systems can aid by classifying the information and in turn helping the human specialists. The computer aided identification technique uses artificial intelligence techniques such as pre-processing, segmentation, feature extraction and classification for decoding the ultimate results which are similar to the other AI-based systems.

The need for managing a person's life with high exactness leads to the introduction of automated detection systems which also guarantees the improvement in diagnosis reducing the adverse after effects of bogus interpretations. It is very evident that twofold interpretation of medical images may lead to an increased probability of cancer detection.

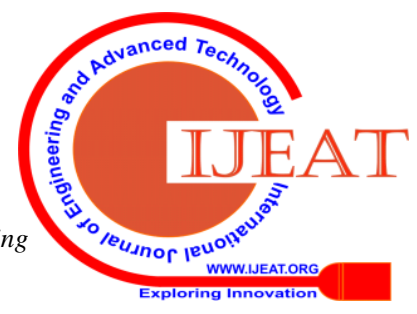




\section{Prediction of Breast Cancer by Segmenting the Image from Mammography using Neural Network Classifier}

Increasing cost of double reading makes it very expensive for patients and hence the dependency on smart software which makes it easier and efficient.On account of the sizable measure of suffers in medical consideration and furthermore the demand for nonstop perception of the above mentioned scenarios, numerous methods of machine-driven demonstrative frameworks are created of late in thisendeavour to unwind this disadvantage.

In similar technical scenario, the quantitative feature detection is done by remodelling the qualitative diagnostic system.

Pixel intensity and a few anatomical alternatives are used to identify the breast resonance images. As of now the lack of widely accepted methods necessitates automatic and reliable ways for cancer detection. The incorrect identification from the breast cancer images can be overcome by using the probabilistic neural network.

\section{EXISTING SYSTEM}

Early discovery of breast cancer through screening and diagnostic systems will expand treatment decisions and survival ratesInterference of humans in the screening techniques leads to the suspicious variations and high level of mistakes because of which there is redundancy in the biopsies done on benign lesions bringing about high burdens to patients.

Wrong interpretation of mammograms will lead to a lot of loss to the patients. The False negatives in the diagnosis leads to a major downside in the treatment. On the other hand early discovery will downsize treatment level, time and fruitful to a decent degree.

A prime reason behind these mistakes is the truth that radiologists depend upon visual examination leading in missing out the important details in figuring out the oversized range of Mammograms. The existing system uses various concepts such as threshold based segmentation, clustering methods, principal component analysis, and co-occurrence matrix.

\section{A. Block Diagram}

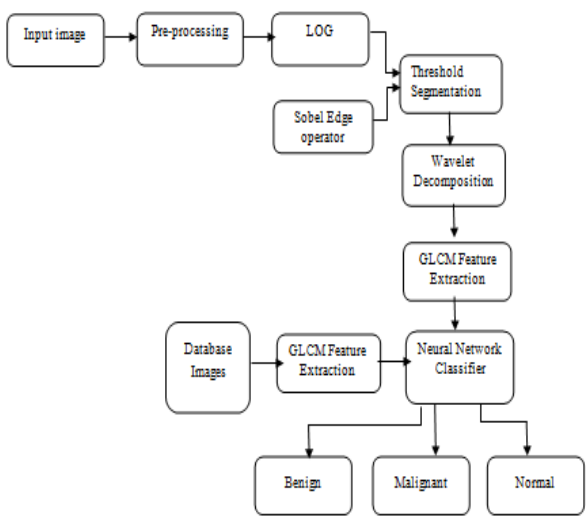

Fig 1: Block Diagram

\section{PROPOSED SYSTEM OVERVIEW}

- Based on segmentation and neural network, the system classifies the image using various methodologies.

- The probabilistic neural system and the DT-CWT are used to group and deduce the property respectively after the image segmentation is done.

- The threshold detection method is utilized for the classification of input image. The CWT channel isolates features and defines the upper limit of the image.

- DTCWT will be used to extract the texture and structural details from a segmented image then the robust local binary pattern will be used to extract the local texture histogram features. It is invariant to local shape and contrast changes.

- Training Models are prepared on normal \& abnormal cases on image classification.

- Training will be given with DTCWT and RLBP to the neural network for automatic classification of images.

- Based on the input vector given, normality/abnormality of the image is classified using the neural network.

\section{A. Sable Edge Operator}

Using computers to try and do image processes have 2 objectives: initial, produce additional appropriate pictures for individuals to look at and determine. Second, we have a tendency to like that computers will mechanically acknowledge and perceive pictures.

The edge of an image is that the most primary component of the picture containing the abundance of internal data. Hence, edge identification is one in all the key investigation works in picture process.

The present picture edge identification procedures are for the most part differential administrator strategy and high-pass filtration. Complications are there due to differential and gradient edge detection methodologies which lead to dissatisfaction. The renowned operators like Sobel, Prewitt, Roberts and Laplacian don't have effective anti-noise performance due to them being delicate to noises.

Despite calculations being complex, the proposed Operators like the log and canny edge detectorsutilize Gaussian function to smooth\& do convolution to the native image.

Edge detection method is exceptionally great due to its anti-noise performance being extraordinary.

The native image brightness is the quality of edge which has a variety of pixels containing the step or roof of the gray price.

A gray value changes to another gray value rapidly and creates a buffer space which is seen in a gray profile. Edgecan be deduced from objects and backgrounds, objects and objects, primitives and primitives. The discontinuing gray projects the object edge. Therefore the edge can be detected by comparing the variation in gray value of one image to the other image which can also be implied as local edge detector. 
Edge detection first utilises the edge enhancement operator in projecting the edges of the image followed by setting the limits and layout of picture elements. The two quantities of Edge detection involves extracting the edge point setand addingthe restructured edge point sets into lines.Sobel edge Operator being a gradient operator gives a smoothing impact to the random noises in the picture brought about by the differential of two rows/two columns and hence edge components are made to look thick and brilliant by upgrade on two sides. The foremost byproduct of digital image relies on the scope of twodimensional gradient estimation in generating the primary by-product or a zero-crossing point and calculates the first order or second order vertical and horizontal gradient values of the image.

The soft threshold wavelet de-noising and Sobel operator are combined so as to:

1. Calculate the edge of the image by forecasting the modulus maxima on angular direction.

2. Avoid difficulty to conclude at the peak value of primary derivative due to mixing up of noise points and important signals

\section{Bi-clustering}

Any abnormality in the image can be deduced by the bi-clustering algorithm. Coexistent row column cluster is executed in this algorithm. This algorithm is employed in some application fields like coclustering, bi-dimensional cluster, two-mode cluster and mathematical space cluster.

$\mathrm{Bi}$-clustering is a very important technique in two-way information analysis by identifying patterns and correlating genes that aids in selection of refined particulars.This helps in cancer detection to obtain accurate results.

\section{THRESHOLD ESTIMATION}

The two directional decomposition is used to find the abnormal region through a threshold limit. The threshold is defined as,

$\mathbf{t}=2 .{ }^{\wedge} \log \left(\operatorname{abs}\left(\mathbf{c}_{\max }\right)\right)$

Where,

$\mathrm{C}_{\max }$ - maximum coefficient of coarse details

Segmentation is performed based on,

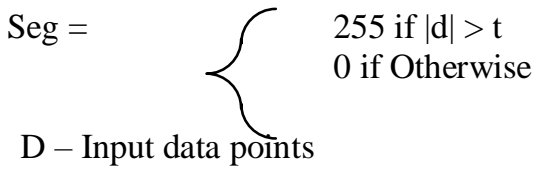

\section{DUAL-TREE COMPLEX WAVELET TRANSFORMS (DT-CWT)}

Thedual-tree complex wavelet transform (DT-CWT) is the improved version of discrete wavelet transform (DWT) with respect to pivotal aspects as it isshift invariant and selective in higher levels. The $2 d$ for $d$ dimensional signals is considerably less than the nondecimatedDWT. The multidimensional (M-D) dual-tree CWT which is computationally efficient works on the separable filter bank (FB).The dual tree transforms helps in designing the complex wavelets throwing up a spread of applications in signal and image processing.

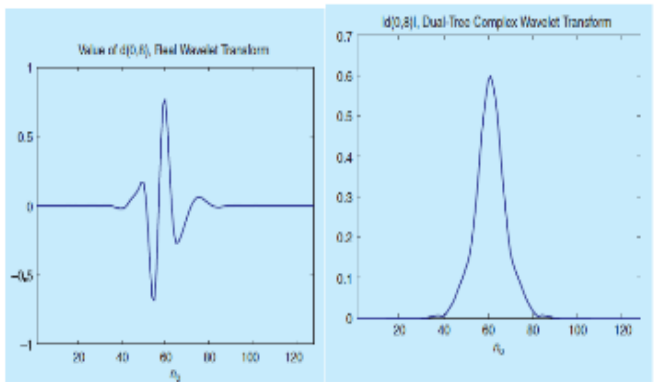

Fig 2: Real-Valued Discrete Wavelet Transforms and Filters Banks wavelet coefficient values

The large and small wavelet coefficients are created by original DWT.Nearness to the edge is directly associated to the coefficientscultivated by the CWT. Here, a step edge at $n=n o, x(n)=u(n-n o)$ is the test signal. The figure shows the value of the wavelet coefficient $d(0,8)$ (the eighth coefficient at stage 3 ) in "real-valued discrete wavelet transforms and filters banks". The conventional real DWT is used to calibrate the real coefficient $d(0,8)$ at the top panel.The complex coefficient $(0,8)$ is calibrated using the dual-tree CWT at the lower panel.

\section{PROBABILISTIC NEURAL NETWORKS}

Biological neuron's activities are rarely supported by neural system. Neural system was the talk of the town in the twentieth century.It really sounds extra energizing than a specialized depiction like "a system of weighted, added value with nonlinear functions". Having said that, the neural systemmeasures from "thinking machines" or "artificial minds". 100 neurons are required for artificial neural system. The nervous system in humans iscomprised of around 3x1010 neurons.

Frank Rosenblatt presented the first perceptron model in 1958 which has three unique layers, (1) "retina" which distributes input to the second layer, (2) "association units" that mixes the inputs with weights and triggers a threshold step operate that feeds to the output layer, (3) "the output layer" which mixes the values. Stage works inside the neuron caused a lot of trouble to the mentors. The basic shortcomings of perceptron's were perceivedin 1969 by Marvin Minsky and Jane Seymourand this faded the interest in perceptron's eventually.

"Learning internal representations by error propagation" was published by David Rumelhart, Geoffrey Hinton and Ronald Williams in 1986 recreated an interest in neural networks. Perceptron's step functions was overcome by the multilayer neural network developed by the above mentioned which utilized nonlinear however differentiable transfer functions. An effective coaching algorithm rule was also invented by them.

Neural system is the best tool for recognition and discrimination.Best outputs are obtained based on the selection of the best algorithm. By mentioning the above; we don't have any verified procedure to

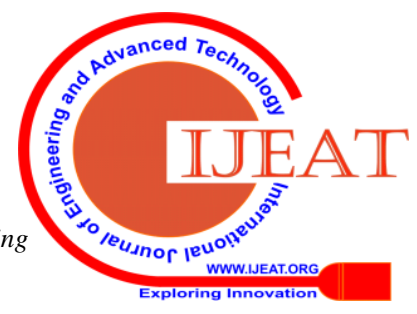




\section{Prediction of Breast Cancer by Segmenting the Image from Mammography using Neural Network}

Classifier

attempt that. The best gratitude to attempt this is to settle on what's relied upon to be suitable in accordance with our past skill at that point to grow or contract the neural system measure until we obtain a suitable output. Diverse sizes for the neural system were attempted using MATLAB.

\section{ADVANTAGES AND DIS-ADVANTAGES OF PNN NETWORKS}

1. Accurate results can be generated by PNN networks.

2. The training of a multilayer perceptron network is much slower than a PNN network.

3. PNNnetworks are relatively imperceptive to outliers (wild points).

4. PNN networks works on Bayes optimal classifier.

5. Accuracy of results from a PNN network is far greater than a multi-layer perceptron network. Accommodation of model takes a large storage space in PNN networks

6. The classification of new cases is much faster in multilayer perceptron than in a PNN network.

\section{DIGITAL IMAGE PROCESSING}

Digitization of the image is the process of crumbling it to a series of numbers, where each number depicts the value of brightness at a specified location or simply a point, technically termed as a picture element or, simply a pixel. A typical image after digitizing has $512 * 512$ pixels, but this count has been increasing rapidly. Once the image is digitised, it can be performed upon by three operations.

- Point operations: Value of output image pixel depends on a single input pixel.

- Local operations: Value of output pixel is calculated by a chunk of input pixels.

- Global operations: Value of output pixel is contributed by tall the input pixels.

The goal of Digital Image processing is, ultimately to enhance, restore or compress an image tailoring to our needs. For example, if we need a clearer image we can use enhancement techniques. Noise smoothening is one such example of enhancement techniques where a $3 \times 3$ median filter is moved across the noisy image to smoothen it. In this technique, the output pixel value depends not only on one pixel but also on the 8 neighbouring pixels. The median value of the 9 pixels will be the out pixel value.

\section{CONCLUSION}

The project presented that mammographic breast cancer diagnosis using new probabilistic network classifier. Here, bi-clustering based breast abnormal tissues segmentation was proposed and it provided better details about abnormality for texture features extraction. CWT was used here to separate the texture details from a segmented image then robust local binary pattern has used to extract the local texture histogram features. It is invariant to local shape and contrast changes. Probabilistic neural network was used here to classify test image into normal or abnormal based on supervised training and its training time is fast and low complexity. Finally the classifier performance was evaluated and proved that proposed system gives better prediction accuracy then prior methodologies.

\section{FUTURE SCOPE}

This system can be enhanced in the future by computerizing it completely. The algorithms which we are using currently can be fed to the system so that the entire process is automated. This will eventually reduce the errors in the analysis .it will also reduce the human interaction which makes the diagnosis more reliable and accurate.

\section{ACKNOWLEDGMENT}

We would like to thank our guide MS.P.SUGANYA for guiding us in this project despite her busy schedule. We would also like to thank our institution for allowing us to display our knowledge by this project.

\section{REFERENCES}

1. Computer Aided TheragnosisBased On Tumour Volumetric Information In Breast Cancer, Mehrdad J. Gangeh, Senior Member, Ieee, Simon Liu, Hadi Tadayyon, And Gregory J. Czarnota,Ieee 2018

2. S. M. Astley, "Computer-Based Detection And Prompting Of Mammographic Abnormalities," Brit. J. Radiol., Vol. 77, No. Suppl 2, Pp. S194-S200, 2004.

3. J. Grim, P. Somol, M. Haindl, And J. Dane`s, "Computer-Aided Evaluation Of Screening Mammograms Based On Local Texture Models," Trans. Img. Proc., Vol. 18, Pp. 765-773, Apr. 2009.

4. X. Gao, Y. Wang, X. Li, And D. Tao, "On Combining Morphological Component Analysis And Concentric Morphology Model For Mammographic Mass Detection," Ieee Trans. Inf. Technol. Biomed., Vol. 14, No. 2, Pp. 266-273, Mar. 2010.

5. M. Sameti, R. Ward, J. Morgan-Parkes, And B. Palcic, "Image Feature Extraction In The Last Screening Mammograms Prior To Detection Of Breast Cancer," Ieee J. Sel. Topics Signal Process. Vol. 3, No. 1, Pp. 46 -52, Feb. 2009.

6. N. Eltonsy, G. Tourassi, And A. Elmaghraby, “A Concentric Morphology Model For The Detection Of Masses In Mammography," Ieee Trans. Med. Imag., Vol. 26, Pp. 880-889, Jun. 2007

7. M. Heath, K. Bowyer, D. Kopans, R. Moore, And W. P. Kegelmeyer, "The Digital Database For Screening Mammography," In Proc. 5th Int. WorkshopDigital Mammography, 2001, Pp. 212-218.

8. M. Fraschini, "Mammographic Masses Classification: Novel And Simple Signal Analysis Method," Electron. Lett., Vol. 47, Pp. 14$15,2011$.

9. M. Hussain, S. Wajid, A. Elzaart, And M. Berbar, "A Comparison Of Svm Kernel Functions For Breast Cancer Detection,” In Proc. 8th Int. Conf. Comput. Graph., Imag. Vis., 2011, Pp. 145-150.

\section{AUTHORS PROFILE}

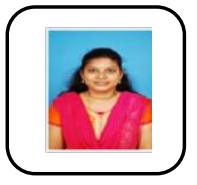

Ms.P.Suganya Assistant Professor (O.G), Area of Interest: Database

Affiliation: Department of Computer Science and Engineering, Ramapuram Campus, SRM Institute of Science and Technology (formerly known as SRM University) 
Ms. Sowmya Ramanathan,Student ,Area of interest: cloud computing, C programming, networking and database

Affiliation: Department of Computer Science and Engineering, Ramapuram Campus, SRM Institute of Science and Technology (formerly known as SRM

University)

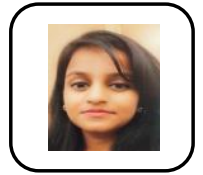

University)

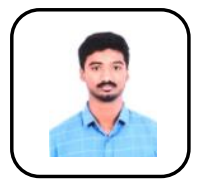

Ms. Prachi

,Student ,Area of Interest:

Android app development and angular

Affiliation: Department of Computer Science and Engineering, Ramapuram Campus, SRM Institute of Science and Technology (formerly known as SRM

Mr. CHVN Anirudh,Student, Area of interest: java programming, android development

Affiliation: Department of Computer Science and Engineering, Ramapuram Campus, SRM Institute of Science and Technology (formerly known as SRM University)

Mr. A.Yogendra Reddy,Student ,Area of interest: java programming, android development

Affiliation: Department of Computer Science and Engineering, Ramapuram Campus, SRM Institute of Science and Technology (formerly known as SRM

University) 